\title{
REVIEW
}

\section{ABDOMINAL WALL AUGMENTATION TECHNIQUES USED IN COMPLEX POSTINCISIONAL HERNIAS REPAIR}

\author{
Roxana Ristea ${ }^{1}$, D. Ion ${ }^{1,2}$, Alexandra Bolocan, R. Palade ${ }^{1,2}$ \\ ${ }^{1}$ The University of Medicine and Pharmacy Carol Davila, Bucharest \\ ${ }^{2}$ Emergency University Hospital of Bucharest
}

Corresponding author: Roxana Ristea Email: roxristea@yahoo.com

\begin{abstract}
One of the most common unwanted consequences of abdominal surgery is the development of an incisional hernia. It occurs in up to $20 \%$ or more of laparotomies. This narrative review aims to put together abdominal wall augmentation techniques used in complex postincisional hernias repair. The research was based on the analysis of the articles in the Scopus, PubMed and WebofScience databases. Due to the high surgical site occurrence rates and recurrence rates, the classic anterior components separation technique without mesh and the modified anterior components separation technique should no longer be performed. Instead of those techniques, endoscopic and perforator sparing anterior components separation techniques are considered better alternatives. The classical posterior components separation techniques and posterior components separation techniques with transversus abdominis muscle release produce better findings than the classic and modified anterior components separation techniques. At present, further data are necessary to assess the role of laparoscopic and robot-assisted posterior components separation technique with TAR.
\end{abstract}

KEYWORDS: abdominal wall augmentation, complex incisional hernia

\section{INTRODUCTION}

One of the most common unwanted consequences of abdominal surgery is the development of an incisional hernia. It occurs in up to $20 \%$ or more of laparotomies [1], [2]. Complex defects of the abdominal wall may also be caused by infection, trauma or tumor resection [3]. Complex and recurrent defects of the abdominal wall represents a challenging surgical and a socio-economic problem due to the time and material expenses [2]. Besides the aesthetic disfigurement those defects may also lead to functional consequences with poor protection of the intra-abdominal viscera [4]. Various surgical approaches can be used in hernia repair in an open fashion or minimally invasive laparoscopic or robotic techniques.

Mesh herniorrhaphy was demonstrated to be superior to suture repair alone, with a recurrence rate of $32 \%$ compared with $63 \%$ in long-term follow-up, in spite of the advances made in mesh materials, surgical technique and perioperative care [1].

Regardless the approach, the tensionfree repair remain the basis of modern herniology [5].

\section{MATERIALS AND METHODS}

The research was based on the analysis of the articles in the Scopus, PubMed and 
WebofScience databases. The search used the keywords: "postincisional hernia" AND "wall augmentation", filtered by language (English) and year of publication.

\section{RESULTS AND DISCUSSIONS}

\section{Open surgery}

Open surgery remains the most common approach for incisional hernia repair, accounting up to $75 \%$ of cases [6]. This approach involves an incision directly over the hernia defect, treatment of the sac content and repositioning of the content in the abdominal cavity followed by closure of the fascial defect with or without a mesh reinforcement. This approach is useful in very small or very large hernias that cannot be solved in a minimally invasive manner. Suture repair can be appropriate for small primary hernias with defects up to $2-3 \mathrm{~cm}$, with a recurrence rate lower than $10 \%$ [7], [8]. Any defect larger than $2-3 \mathrm{~cm}$ needs a mesh reinforcement.

Compared with minimally invasive approaches, open techniques have a higher rate of postoperative surgical site infections (SSI) and surgical site occurrence (SSO) though this varies regarding the technique and mesh position [9], [10].

Regarding the cases of complex hernias, the classical approaches (sublay, onlay) do not suit to be used because of the risk of intraabdominal hypertension development followed by organ dysfunction and organ failure [11]. An inlay technique can obtain a tension free repair but it is followed by a postoperative diastasis recti abdominis and in time we obtain a dysfunctional abdominal wall, leading to abdominal recti atrophy [5].

Currently, there are relatively few techniques that can solve the problems mentioned above. Any variant is based on the use of separation technique of the abdominal wall components that separate the musculofascial layers of the abdominal wall to allow sliding of the muscular component back to midline and decrease tension on the closure. Among these techniques there distinguish anterior components separation techniques and posterior components separation techniques.
1. Release of the rectus sheath was first described by Jean Rives [12] though not termed as "component separation". The technique involves the opening of the rectus sheath and separation of the posterior sheath from the rectus muscle, continuing laterally with the retromuscular space dissection to the linea semilunaris and extended superiorly and inferiorly to allow mesh overlap of the hernia defect. This dissection leads to a posterior sheath mobility towards medial border up to $6 \mathrm{~cm}$ that makes possible to close the posterior plan in a tension-free manner. After the posterior sheath closure, a mesh is placed in a sublay retromuscular manner and the anterior midline fascia is closed over the mesh [13].

In large-sized hernia defects, this procedure appears to be insufficient to obtain the abdominal wall closure in a tension-free manner.

\section{Anterior components separation technique} was described by Oscar Ramirez as a response to this large-size defects herniorrhaphy problem; this surgical approach allows the closure of defects up to $20 \mathrm{~cm}$, by gaining approximately $8-10 \mathrm{~cm}$ medialization of the rectus sheath bilaterally [14].

The technique, known since 1990 is a basic procedure for components separation and represent a combination of techniques performed both in the middle and the lateral segments of the abdominal wall. The basic principle include bilateral midline to lateral dissection in a subcutaneous plane adjacent to the anterior rectus sheath until the semilunaris linea is reached followed by the transection of the aponeurosis of the external oblique muscle. The incision is made $2 \mathrm{~cm}$ laterally from the rectus sheath, extending from the inguinal ligament to the level of costal margin. The external oblique muscle is separated from the internal oblique muscle as far laterally as possible avoiding any injury to the internal oblique fascia or muscle and to the segmental innervations of the rectus muscle. The dissection between external and internal oblique muscles is done in a relatively avascular plane and is continued toward the level of midaxillary line or until the lumbar veins appear [15]. The technique enables to mobilize the rectus abdominis medially, the muscles being placed in 
their physiological position. Using this approach, the surgeon gains mobilization of 4 $\mathrm{cm}$ in the upper abdomen, $8 \mathrm{~cm}$ in the umbilical area and $3 \mathrm{~cm}$ in the lower abdomen [13]. According to other sources, the mobility is 5$10 \mathrm{~cm}, 10-15 \mathrm{~cm}$ and $3-8 \mathrm{~cm}$ in these abdominal areas, respectively [16]. If further mobilization is needed, the posterior rectus sheath may also be incised longitudinally and dissected in a Rives - Stoppa manner.

Once released from the external oblique muscle and its fascia, the compound flap of rectus muscle and the attached internal oblique and transversus abdominis muscle is advanced medially. The closure is done with a single layer of heavy nondissolvable monofilament suture [17].

The procedure can be done with or without the reinforcement of the fascia with mesh, the tisular method experiencing a recurrence rate between 10-22\% [18].

Though effective for defect closure, the Ramirez technique carries significant morbidity, with nearly half of patients experiencing wound complications [19]. The main drawback of the anterior separation hernia repair is the necessity to separate large dermal-fat grafts that increase the SSI up to $26-63 \%$ [20]

\section{Posterior components separation}

Described by Carbonell et al in 2008 [21] the technique involve a large mesh implant use. The authors recommend a midline laparotomy that treats the herniar content. The retromuscular space is developed by incising the posterior rectus sheath and dissecting the muscle anteriorly. Once the lateral-most edge of the rectus sheath is reached, the posterior rectus sheath is incised, dividing the posterior aponeurotic sheath of the internal oblique muscle. This allows entering the plane between the internal oblique and transversus abdominis muscles. Below the semicircular line of Douglas where the posterior rectus sheath ends, we enter the space ventral to transversalis fascia. For hernia defects located in the proximity of xiphoid process is necessary a cephalad dissection into the retroxiphoidal space [22]. The posterior rectus sheath is reapproximated in the midline with a running absorbable suture. [23]. The mesh is placed in the retromuscular space and secured with transabdominal permanent sutures and is covered by reapproximation of the anterior sheath in the midline.

The posterior components separation technique acquire two goals in hernia repair first, medial mobilization of the transversus abdominis muscle with accompanying posterior rectus sheath leads to less tension and complete closure of the posterior layer, creating a safe space for the mesh. Second, medial mobilization of the internal and external obliques with accompanying anterior rectus sheath allows a complete closure of the anterior abdominal wall above the prosthesis, lowering the risk of deep prosthetic infections [24]. One of the major advantages and in the same time critics of Ramirez anterior component separation is the lack of large subcutaneous flap elevation, reducing the risk of seroma formation.

Due to the fact that anterolateral portion of the abdominal wall and the rectus muscle are supplied by the anterior rami of the 7 th-12th thoracic nerves, which course between the internal oblique and transversus muscle before they penetrate the posterior rectus sheath, the course of the first lumbar nerve and lateral cutaneous nerve branches between the internal oblique and transversus muscle, a careful dissection may be conducted. Sectioning one or more of these nerves can lead to rectus paralysis and abdominal wall weakness. Since retromuscular hernia repairs occasionally sacrifice the distal-most aspect of these nerves, paralysis is much less likely.

\section{Transversus abdominis muscle release (TAR)}

Described in 2012 by Novitsky et al., this technique represents a posterior components separation method which involves division of the transversus abdominis muscle and fascia with dissection of the plane between the muscle and peritoneum laterally [25].

The dissection begins with a median laparotomy with complete excision of the previous scar, dissection of the hernia sac and adhesiolysis. Creation of the retrorectus space is initiated at the level of the umbilicus through an incision made on the posterior sheath $0,5-1 \mathrm{~cm}$ apart from the medial edge [26]. Laterally the dissection continue to linea semilunaris, visualizing the junction between the posterior and anterior rectus sheath, with visualization of 
the epigastric vessels and the branches of thoracoabdominal nerves penetrating the posterior rectus sheath. Care must be taken to avoid injury of these elements. The exposure of transversus abdominis muscle is obtained by an incision made $0,5 \mathrm{~cm}$ medial to the anteriorposterior rectus sheath junction, medial to the neurovascular elements. This incision is placed in the upper third of the abdomen. The muscle is then divided vertically along its entire length along the medial margin. The muscle is easier to identify at this level, being well developed, his volume regressing in the distal two thirds of the abdominal wall where only its tendinous component can be identified and divided.

This step allows entrance in the space between transversalis fascia and the divided transversus abdominis muscle.

The retromuscular plane is obtain by blunt or sharp dissection and can be extended cranial to the retroxiphoid plane and caudal in the space of Retzius with identification of bilateral Cooper's ligaments and pubic symphisis. Parietalization of the cord or resection of the round ligament extends the dissection in the pro-peritoneal space of Bogros and the myopectineal triangles.

Laterally, the dissection can be extended as far as the psoas muscle, its lateral border being used as a landmark, but usually the posterior axillary line is the main anatomic landmark used for lateral dissection.

After the dissection is complete on both sides the posterior rectus sheath is closed in the midline with a running monofilament suture. The mesh is then placed in this retromuscular, pro-peritoneal space created, overlapping the defect minimally to anterior axillary line, subxiphoid space and $2-4 \mathrm{~cm}$ below the pubic simphisis [26]. The anterior rectus sheath is then reapproximated in the midline, to restore the linea alba.

This technique minimizes the subcutaneous dissection, allows myofascial release to decrease the midline tension and permits wide mesh coverage in a well vascularized compartment, separated from the visceral cavity [27], many authors considering it as the gold standard for open ventral hernia repair[28].

\section{Minimally invasive approaches}

\section{Endoscopic anterior component separation}

Reported by Rosen et al., the operation involves trocar placement just below the costal margins lateral to the rectus abdominis muscle. "The subcutaneous tissue is bluntly dissected exposing the external oblique aponeurosis. The aponeurosis fibers are split in their natural orientation, exposing the internal oblique muscle. The space between the internal and external oblique is created using a hernia balloon dissector. A structural balloon port is then place in this space created to maintain the insufflation pressure of $12 \mathrm{mmHg}$. The dissection is carried away with the tip of a $10 \mathrm{~mm}, 30$ degree laparoscope. Two additional $5 \mathrm{~mm}$ ports are then placed and the external oblique is released from the costal margin to the inguinal ligament using coagulation scissors or ultrasonic scissors. The process is continues bilateral. If additional release is deemed necessary, the posterior rectus sheath is incised in the midline.’'[29].

2. Anterior component separation technique with perforator preservation using balloon dissection

"Fascial separation is done through separate inguinal incisions. After incising the external oblique aponeurosis as in standard inguinal hernia repair, the balloon dissector is placed between the external and internal oblique muscles, advanced cephalad, and inflated. The lateral border of the rectus muscle acts as an anatomical barrier and forces the balloon to expand laterally, creating the necessary space. With headlamp illumination and a narrow retractor, a sponge forceps completes the fascial separation. Ultrasonic shears are then used to incise the elevated external oblique aponeurosis and the muscular portion found more cephalad. After fascial release has been done bilaterally, the midline scar is excised and minimal skin flaps are raised to free the hernia sac, thus preserving the periumbilical perforator vessels. The posterior rectus sheath is incised from within the midline incision. Midline closure is done as described for the "classic" anterior CST" [17]. 
Laparoscopic posterior component separation technique and transversus abdominis release

The technique was presented by Belyansky et al. [30]. "Typically three ports are placed bilaterally. After reduction of the hernia content and adhesiolysis, the posterior rectus sheath is released approximately 0.5 to $1 \mathrm{~cm}$ lateral to the edge of the defect and linea alba. The incision in the posterior rectus sheath is performed along its whole length from cephalad to caudal direction. Hook electrocautery combined with laparoscopic scissors sharp dissection are used to achieve this release, which exposes the posterior portion of the rectus abdominis muscle. When performed appropriately it can allow up to $3 \mathrm{~cm}$ of medial mobilization of the edge of the defect. Once the posterior rectus sheath is released, atraumatic graspers are used to retract the free edge of posterior rectus sheath medially to facilitate blunt dissection in the retrorectus space laterally toward the linea semilunaris. The neurovascular bundles that travel between the internal oblique and transversus muscles and then perforate the rectus abdominis muscle are identified laterally in this space and are preserved to prevent rectus defunctionalization and atrophy. With preservation of the neurovascular bundle about $0.5-1 \mathrm{~cm}$ medial to the anterior/posterior rectus sheath junction, the posterior rectus sheath is incised to expose the underlying transversus abdominis muscle. Hook electrocautery is then used to elevate the transversalis muscle fibers and cauterize them. As the transversus fibers are cut the posterior layer of glistening transversalis fascia is exposed. Transversalis release is performed in this fashion in a cephalad to caudal direction. Blunt dissection just posterior to the transversus muscle and superficial to the transversalis fascia is performed and is carried past the midaxillary line. Unilateral TAR can achieve up to $7 \mathrm{~cm}$ of fascial medial mobilization. After closure of the posterior rectus sheath in a running fashion, the mesh is placed behind the muscles and fixed. The anterior fascial defect is then sutured "upside down" [30].

\section{Robotic surgery}

The robotic approach is a more recent achievement in the field of herniorrhaphy, providing several advantages including enhanced 3D visualization, tremor elimination and articulating instruments. Essentially, the robotic approach reproduces the laparoscopic techniques with the advantage of an easier intracorporeal hernia defect closure and the lack of multiple incisions necessary for transcutaneous closure [31]. Compared with the open approach for the retromuscular hernia repair, the technique encounters similar incidence of SSI, equivalent recurrence rate and similar direct hospitalization cost, but with a shorter hospital length of stay [32].

\section{Advantages and limits of components separation techniques}

\section{Anterior component separation}

Since the anterior component separation gains sufficient mobility of the musculofascial flaps that can solve hernia defects up to $20 \mathrm{~cm}$, the next question that arise is if it should be used with or without a mesh placement in herniorrhaphy?

The main criticism of the technique is the large skin and subcutaneous tissue mobilization that creates a large wound surface area predisposed to hematoma and seroma formation and infection. Another drawback of the method is that the mobilization of the skin and subcutaneous tissue endangers the blood supply of this area, which can lead to skin necrosis and dehiscence of the surgical incision [33]-[37].

Open anterior components separation technique with mesh compared with open anterior components separation technique alone appear to be associated with fewer hernia recurrences and overall complications [38].

The systematic review by Deerenberg at al. based on seven studies of herniorrhaphy with classic components separation technique, without mesh, accounts postoperative complications in almost $50 \%$ of the patients and a recurrence rate of $16 \%$ [39].

In a systematic review of autologous tissue repair of large abdominal wall defects, component separation technique was associated with high morbidity rate of $24 \%$ and a recurrence rate after one year of $18,2 \%$ [40].

Similar results were obtained by different authors who conducted prospective 
studies and compared them with the literature data [41]-[43], concluding that repair of large hernias without a mesh placement coincides with a high recurrence rate [43], [44].

An expert consensus guided by systematic review of abdominal wall hernia management recommends the avoidance of component separation technique without mesh reinforcement [45].

Regarding the approach method - open or endoscopic, the guidelines recommend the endoscopic techniques [45] based on systematic reviews that point SSO in $21.4 \%$ of cases vs. $20.3 \%$ for open vs. endoscopic anterior components separation, respectively a recurrence rate of $11.9 \%$ vs. $7 \%$ [46].

\section{Posterior component separation technique}

After the development and implementation of posterior components separation technique in the classical manner and transversus abdominis release, the next question that arises is their efficiency compared with classical anterior separation technique.

In a case series of 20 patients with classical posterior components separation technique, three developed wound complications (15\%) and one recurrence (5\%) after a mean 12 month follow-up [21].

A retrospective comparative study [47] highlight the fact that wound complications occurred significantly more in open anterior versus open posterior components separation technique ( $48.2 \%$ vs. $25.5 \%$ ) with a higher recurrence rate in the anterior component separation group ( 14.3 vs. $3.6 \%$ ).

A recent systematic review by Cornette et al. [46] comparing 22 studies with 1348 cases of open anterior approach and 8 studies with 761 cases for posterior approach pointed out a rate of surgical site occurrence of $21.4 \%$ for the anterior approach and $20.3 \%$ for posterior approach. The recurrence rates were $11.9 \%$ vs. $5.25 \%$.

\section{CONCLUSIONS}

Due to the high surgical site occurrence rates and recurrence rates, the classic anterior components separation technique without mesh and the modified anterior components separation technique should no longer be performed.

Instead of those techniques, endoscopic and perforator sparing anterior components separation techniques are considered better alternatives.

The classical posterior components separation techniques and posterior components separation techniques with transversus abdominis muscle release produce better findings than the classic and modified anterior components separation techniques.

At present, further data are necessary to assess the role of laparoscopic and robotassisted posterior components separation technique with TAR.

\section{REFERENCES}

[1] J. W. A. Burger et al., "Long-term follow-up of a randomized controlled trial of suture versus mesh repair of incisional hernia," in Annals of Surgery, 2004.

[2] B. K. Poulose et al., "Epidemiology and cost of ventral hernia repair: Making the case for hernia research," Hernia, 2012.

[3] S. Adekunle, N. M. Pantelides, N. R. Hall, G. Surg, R. Praseedom, and C. M. Malata, "Indications and Outcomes of the Components Separation Technique in the Repair of Complex Abdominal Wall Hernias: Experience From the Cambridge Plastic Surgery Department," Eplasty, 2013.

[4] T. Abulezz, "Repair of recurrent midline abdominal wall dehiscence using the components separation technique," Journal of Plastic, Reconstructive and Aesthetic Surgery. 2008.

[5] V. V. Parshikov and V. I. Loginov, "Components separation technique in treatment of patients with ventral and incisional hernias (Review)," Sovremennye Tehnologii v Medicine. 2016.

[6] C. Tsui, R. Klein, and M. Garabrant, "Minimally invasive surgery: National trends in adoption and future directions for hospital strategy," Surg. Endosc., 2013.

[7] M. T. Nguyen et al., "Comparison of outcomes of synthetic mesh vs. suture repair of elective primary ventral herniorrhaphy: A systematic review and meta-analysis," JAMA Surg., 2014.

[8] J. L. Porrero et al., "Umbilical hernia repair: Analysis after 934 procedures," in American Surgeon, 2015.

[9] I. K.M.F. et al., "Comparison of laparoscopic and open repair with mesh for the treatment of ventral incisional hernia: A randomized trial," Arch. Surg., 2010. 
[10] C. S. Hwang, K. A. Wichterman, and E. J. Alfrey, "Laparoscopic Ventral Hernia Repair is Safer than Open Repair: Analysis of the NSQIP Data1,” J. Surg. Res., 2009.

[11] M. Schein, "The abdominal compartment syndrome," in Schein's Common Sense Emergency Abdominal Surgery (Second Edition), 2005.

[12] R. Stoppa, D. Louis, P. Verhaeghe, X. Henry, and J. P. Plachot, "Current surgical treatment of post-operative eventrations," Int. Surg., 1987.

[13] P. Bogetti, F. Boriani, G. Gravante, A. Milanese, P. M. Ferrando, and E. Baglioni, "A retrospective study on mesh repair alone vs. mesh repair plus pedicle flap for large incisional hernias.," Eur. Rev. Med. Pharmacol. Sci., 2012.

[14] O. M. Ramirez, E. Ruas, and A. L. Dellon, "'Components separation' method for closure of abdominal-wall defects: An anatomic and clinical study," Plast. Reconstr. Surg., 1990.

[15] L. Heller, C. Chike-Obi, and A. S. Xue, "Abdominal wall reconstruction with mesh and components separation," Seminars in Plastic Surgery. 2012.

[16] D. H. Shell IV, J. de la Torre, P. Andrades, and L. O. Vasconez, "Open Repair of Ventral Incisional Hernias," Surgical Clinics of North America. 2008.

[17] J. M. Clarke, "Incisional hernia repair by fascial component separation: Results in 128 cases and evolution of technique," Am. J. Surg., 2010.

[18] J. H. Ko, E. C. Wang, D. M. Salvay, B. C. Paul, and G. A. Dumanian, "Abdominal wall reconstruction: Lessons learned from 200 'components separation' procedures," Arch. Surg., 2009.

[19] E. M. Pauli and M. J. Rosen, "Open ventral hernia repair with component separation," Surgical Clinics of North America. 2013.

[20] R. Gonzalez, R. D. Rehnke, A. Ramaswamy, C. D. Smith, J. M. Clarke, and B. J. Ramshaw, "Components separation technique and laparoscopic approach: A review of two evolving strategies for ventral hernia repair," in American Surgeon, 2005.

[21] A. M. Carbonell, W. S. Cobb, and S. M. Chen, "Posterior components separation during retromuscular hernia repair," Hernia, 2008.

[22] J. Conze, A. Prescher, K. Kisielinski, U. Klinge, and V. Schumpelick, "Technical consideration for subxiphoidal incisional hernia repair," Hernia, 2005.

[23] V. A. Samartsev, A. G. Kuchumov, and V. A. Gavrilov, "Sutures in abdominal surgery: Biomechanical study and clinical application," Cent. Eur. J. Med., 2014.

[24] S. Petersen, G. Henke, L. Zimmermann, G. Aumann, G. Hellmich, and K. Ludwig, "Ventral rectus fascia closure on top of mesh hernia repair in the sublay technique," Plastic and Reconstructive Surgery. 2004.

[25] Y. W. Novitsky, H. L. Elliott, S. B. Orenstein, and M. J. Rosen, "Transversus abdominis muscle release: a novel approach to posterior component separation during complex abdominal wall reconstruction.," Am. J. Surg., 2012.

[26] V. Oprea, V. Gheorghe Radu, and D. Moga, "Transversus Abdominis Muscle Release (TAR) for Large Incisional Hernia Repair," Chirurgia (Bucur)., 2016.

[27] W. S. Cobb, J. A. Warren, J. A. Ewing, A. Burnikel, M. Merchant, and A. M. Carbonell, "Open retromuscular mesh repair of complex incisional hernia: Predictors of wound events and recurrence," in Journal of the American College of Surgeons, 2015.

[28] F. P. Albino, K. M. Patel, M. Y. Nahabedian, M. Sosin, C. E. Attinger, and P. Bhanot, "Does mesh location matter in abdominal wall reconstruction? A systematic review of the literature and a summary of recommendations," Plast. Reconstr. Surg., 2013.

[29] M. J. Rosen, J. Jin, M. F. McGee, C. Williams, J. Marks, and J. L. Ponsky, "Laparoscopic component separation in the single-stage treatment of infected abdominal wall prosthetic removal," Hernia, 2007.

[30] I. Belyansky, H. R. Zahiri, and A. Park, "Laparoscopic Transversus Abdominis Release, a Novel Minimally Invasive Approach to Complex Abdominal Wall Reconstruction," Surg. Innov., 2016.

[31] M. V. F. DO AMARAL et al., "Robotic Transversus Abdominis Release (TAR): is it possible to offer minimally invasive surgery for abdominal wall complex defects?," Rev. Col. Bras. Cir., 2017.

[32] A. M. Gonzalez, R. J. Romero, R. Seetharamaiah, M. Gallas, J. Lamoureux, and J. R. Rabaza, "Laparoscopic ventral hernia repair with primary closure versus no primary closure of the defect: Potential benefits of the robotic technology," Int. J. Med. Robot. Comput. Assist. Surg., 2015.

[33] J. N. DiBello and J. H. Moore, "Sliding myofascial flap of the rectus abdominus muscles for the closure of recurrent ventral hernias," Plast. Reconstr. Surg., 1996.

[34] J. B. Lowe, J. R. Garza, J. L. Bowman, R. J. Rohrich, and W. E. Strodel, "Endoscopically Assisted Components Separation for Closure of Abdominal Wall Defects," Plast. Reconstr. Surg., 2003.

[35] K. C. Shestak, H. J. Edington, and R. R. Johnson, "The separation of anatomic components technique for the reconstruction of massive midline abdominal wall defects: anatomy, surgical 
technique, applications, and limitations revisited.," Plast. Reconstr. Surg., 2000.

[36] J. A. Girotto, M. J. Ko, R. Redett, T. Muehlberger, M. Talamini, and B. Chang, "Closure of chronic abdominal wall defects: A long-term evaluation of the components separation method," Ann. Plast. Surg., 1999.

[37] M. Cohen, R. Morales, J. Fildes, and J. Barrett, "Staged reconstruction after gunshot wounds to the abdomen," Plast. Reconstr. Surg., 2001.

[38] W. M. Y. Tong, W. Hope, D. W. Overby, and C. S. Hultman, "Comparison of outcome after meshonly repair, laparoscopic component separation, and open component separation," in Annals of Plastic Surgery, 2011.

[39] E. B. Deerenberg et al., "A systematic review of the surgical treatment of large incisional hernia," Hernia. 2015.

[40] T. S. De Vries Reilingh, M. E. Bodegom, H. Van Goor, E. H. M. Hartman, G. J. Van Der Wilt, and R. P. Bleichrodt, "Autologous tissue repair of large abdominal wall defects,” Br. J. Surg., 2007.

[41] A. Eriksson, J. Rosenberg, and T. Bisgaard, "Surgical treatment for giant incisional hernia: A qualitative systematic review," Hernia. 2014.
[42] N. J. Slater, H. Van Goor, and R. P. Bleichrodt, "Large and complex ventral hernia repair using 'components separation technique' without mesh results in a high recurrence rate," Am. J. Surg., 2015.

[43] T. S. D. V. Reilingh et al., "Repair of giant midline abdominal wall hernias: 'Components separation technique' versus prosthetic repair: Interim analysis of a randomized controlled trial," in World Journal of Surgery, 2007.

[44] E. B. O’Halloran, C. J. Barwegen, J. M. Dombrowski, D. K. Vandevender, and F. A. Luchette, "Can't have one without the other: Component separation plus mesh for repairing difficult incisional hernias," Surg. (United States), 2014.

[45] M. K. Liang et al., "Ventral Hernia Management,” Ann. Surg., 2016.

[46] B. Cornette, D. De Bacquer, and F. Berrevoet, "Component separation technique for giant incisional hernia: A systematic review," American Journal of Surgery. 2018.

[47] D. M. Krpata, J. A. Blatnik, Y. W. Novitsky, and M. J. Rosen, "Posterior and open anterior components separations: A comparative analysis," Am.

$\mathrm{J}$.

Surg.,

2012. 